\title{
Method for finding majorant function estimates required to determine Picard's successive approximations convergence domain
}

\author{
Vera Petelina ${ }^{1, *}$ \\ ${ }^{1}$ Moscow State University of Civil Engineering, Yaroslavskoye shosse, 26, Moscow, 129337, Russia
}

\begin{abstract}
This paper deals with convergence of Picard's successive approximations, which give a solution for perturbed body motion differential equations system through constructing a majorizing linear differential equation. The task is to determine the domain of used variables where these successive approximations converge and to estimate how large the error will be if we take a finite number of approximations. A method for finding majorant function estimates required to determine Picard's successive approximations convergence domain is constructed.
\end{abstract}

\section{Introduction}

The paper [2] gave a representation of the rectangular coordinates constituting regularized velocity and time of perturbed body motion in the second approximation in the form of polynomials in powers of some regularizing variable. It can be done similarly one more time to find the third, fourth and higher approximations, because analytic features of these functions are known. Thus, we obtain an iterative process of finding a solution for the perturbed body motion differential equations system.

\section{Mathematical model}

The paper [3] proved convergence of successive approximations and determined the conditions under which successive approximations converge to an exact solution. Let's consider the following differential equations system:

$$
\frac{d C_{i}}{d u}=\varepsilon\left[f_{i 0}(u)+f_{i 1}\left(u, C_{1}, C_{2}, \ldots, C_{7}\right)\right]
$$

Where: $\varepsilon$-small parameter,

$u$ - independent variable, while $-1<u<1, C_{i}$ in the problem under consideration these are the variations of osculating initial values of the rectangular coordinates $\xi, \eta, \zeta$, constituting regularized velocity $\xi^{\prime}, \eta^{\prime}, \zeta^{\prime}$ instant of time $\tau$.

\footnotetext{
*Corresponding author: verapetelina51@gmail.com
} 
$f_{i 0}(u)$ - right-hand sides of differential equations system with:

$$
\begin{gathered}
\xi=\xi_{0} ; \eta=\eta_{0} ; \zeta=\zeta_{0} ; \xi^{\prime}=\xi_{0}^{\prime} ; \eta^{\prime}=\eta^{\prime} 0 ; \zeta^{\prime}=\zeta_{0}^{\prime} ; \tau=\tau_{0} \\
f_{i 1}\left(u, C_{1}, C_{2}, \ldots, C_{7}\right)=f_{i}\left(u, C_{1}+\xi_{0}, C_{2}+\eta_{0}, \ldots, C_{7}+\tau_{0}\right)-f_{i}\left(u, \xi_{0}, \eta_{0}, \ldots, \tau_{0}\right)
\end{gathered}
$$

We can choose such a $C^{*}$ that the right-hand sides of the differential equations system (1) in the hyperparallelepiped $D:-1 \leq u \leq 1 ;\left|C_{i}\right| \leq C^{*}$ are continuous as per $u$ and will satisfy the generalized Lipschitz conditions (4):

$$
\left|f_{i 1} u, C_{1}^{*}, C_{2}^{*}, \ldots, C_{7}^{*}-f_{i 1} u, C_{1}^{* *}, C_{2} * *, \ldots, C_{7} * *\right| \leq \sum_{j=1}^{7} L_{i j} u \cdot\left|C_{j}^{*}-C_{j} * *\right|
$$

where: $L_{i j} u=\sup \left|\frac{\partial f_{i 1}}{\partial C_{j}}\right|$ in the hyperparallelepiped $D_{u}$ which is the cross section of the hyperparallelepiped $D$ with the plane $u=u_{0}$.

We find a solution for the differential equations system (1) through successive approximations method.

In the $\mathrm{k}^{\text {th }}$ approximation:

$$
\begin{aligned}
& C_{i}^{(k)}(u)=C_{i}^{(1)}(u)+\varepsilon \cdot \int_{-1}^{u} f_{i 1}\left(u, C_{1}^{(k-1)}(u), \ldots, C_{7}^{(k-1)}(u)\right) \cdot d u= \\
& =\varepsilon \cdot \int_{-1}^{u}\left(f_{i 0}(u)+f_{i 1}\left(u, C_{1}^{(k-1)}(u), \ldots, C_{7}^{(k-1)}(u)\right)\right) \cdot d u
\end{aligned}
$$

We form a linear differential equation with variable coefficients:

$$
\frac{d C}{d u}=\varepsilon[P(u)+Q(u) \cdot C]
$$

which is considered in the interval $-1 \leq u \leq 1$.

Auxiliary functions $P(u)$ and $Q(u)$ satisfy the conditions of

$$
\left|f_{i 0} u\right| \leq P u ;\left|L_{i j} u\right| \leq L u ;|Q u| \leq 7 \cdot L u
$$

$(i=1,2 \ldots, 7)$

Solution of the equation (6) is represented by an absolutely convergent series in terms of powers of $u$.

If: $\quad C(u)=\sum_{k=1}^{\infty} \varepsilon^{k} \cdot \tilde{C}^{(k)}(u) ; C(u)=e^{\varepsilon \cdot \int_{-1}^{1} Q(u) \cdot d u} \cdot \varepsilon \cdot \int_{-1}^{1} P(u) \cdot e^{-\varepsilon \cdot \int_{-1}^{u} Q(u) \cdot d u} \cdot d u \leq C^{*}$

Let's find a solution of the equation (6) using successive approximations and then after $l$ of iterations we get:

$$
C^{(l)}(u)=\sum_{1}^{(l)} \varepsilon^{k} \cdot \tilde{C}^{(k)}(u),
$$

where: $\varepsilon^{k} \cdot \tilde{C}^{(k)}(u)=C^{(k)}(u)-C^{(k-1)}(u)$

\section{Method for finding majorant function estimates required to evaluate successive approximations convergence domain}

The paper [3] proved that if inequality (8) was satisfied, the successive approximations, representing a solution for the perturbed body motion differential equations system $C^{(1)}(u)$, absolutely converged. The set task is to estimate the convergence domain of majorizing 
equation resulting solution (6), to do this it is necessary to estimate the quantity $C^{*}$. To estimate the quantity $C^{*}$, it is necessary to estimate the right-hand sides of perturbed body motion differential equations system [2], where the unknown variables are osculating initial values of the rectangular coordinates $\xi, \eta, \zeta$

- constituting the regularized velocity $\xi^{\prime}, \eta^{\prime}, \zeta^{\prime}$

- of regularized acceleration $\xi^{\prime \prime}, \eta^{\prime \prime}, \zeta^{\prime \prime}$

- of instant of time $\tau$, radius-vector $\rho$, and its derivatives $\rho^{\prime}$ and $\rho^{\prime \prime}$ according to an independent regularizing variable $u$ taking into account the assumptions.

To do this, the osculating initial values of rectangular coordinates constituting regularized velocity, regularized acceleration and time of geocentric body motion were added with the terms depending on the parameter $C^{*}$ taken with their scale coefficients; i.e. the terms $C_{i}=\gamma_{i} \cdot C^{*}(i=1,2, \ldots, 7)$, while the coefficients $\gamma_{1}, \gamma_{2}, \gamma_{3}$ are dimensionless, $\gamma_{4}, \gamma_{5}, \gamma_{6}$ are the coefficients with dimension $(\mathrm{km} / \mathrm{sec}) \gamma_{7}$ is the coefficient with dimension $(\mathrm{sec} / \mathrm{km})$. For each function being a part of the right-hand sides of the differential equations system [2], the right and left boundaries, which depend on the quantity $C^{*}$, were estimated; to find the left boundary, we sought to obtain the minimum quantity and the components $C_{i}=\gamma_{i} \cdot C^{*}(i=1,2, \ldots, 7)$ were taken with a negative sign, to find the right boundary, we sought to obtain the maximum quantity. The maximum estimator modulo was chosen as the unknown estimate. Thus, we found fairly rough function estimates, to find which, geometric considerations were mainly used. More accurate estimates of these functions were constructed taking into account the behavior of the functions $x, y, z, x^{\prime}, y^{\prime}, z^{\prime}, \xi^{\prime \prime}, \eta^{\prime \prime}, \zeta^{\prime \prime}, \sigma$ and their derivatives with respect to the variable $\rho$. Let's illustrate the above through the example of finding a rough and more accurate majorant estimate for the function $\sigma$.

$$
\sigma=\frac{\left(\xi^{\prime 2}\right)}{\rho^{2}}+\frac{\left(\eta^{\prime 2}\right)}{\rho^{2}}-\frac{2 \mu}{\rho}=\frac{v^{\prime 2}}{\rho^{2}}-\frac{2 \mu}{\rho}
$$

where $v^{\prime}=v \cdot \rho, v=\sqrt{\dot{\xi}^{2}+\dot{\eta}^{2}}$.

A rough estimate for the function $\sigma$ is written as:

$$
\begin{gathered}
\sigma \leq \sigma_{+}^{*}=\frac{\left(\gamma_{4} \cdot C^{*}\right)^{2}+\left(v^{\prime}+\gamma_{5} \cdot C^{*}\right)^{2}}{\left(\rho-\gamma_{1} \cdot C^{*}\right)^{2}}-\frac{2 \mu}{\rho+\gamma_{1} \cdot C^{*}} \\
\sigma \geq \sigma_{-}^{*}=\frac{\left(v^{\prime}-\gamma_{5} \cdot C^{*}\right)^{2}}{\left(\rho+\gamma_{1} \cdot C^{*}\right)^{2}}-\frac{2 \mu}{\rho-\gamma_{1} \cdot C^{*}}
\end{gathered}
$$

To find a more accurate estimate for the function $\sigma$,

let's determine $\frac{\partial \sigma}{\partial \rho}=-\frac{2 v^{\prime 2}}{\rho^{3}}+\frac{2 \mu}{\rho^{2}}$

$$
\frac{\partial \sigma}{\partial \rho}=0 \quad \text { with } v^{\prime}=\sqrt{\mu \cdot \rho} \text {, hence } \rho=\frac{v^{\prime 2}}{\mu} \text { and } \sigma\left(\frac{v^{\prime 2}}{\mu}\right)=-\frac{\mu^{2}}{v^{\prime 2}} \prec 0 \text {. }
$$

This is the minimum of the function $\sigma(\rho)$, since with $\rho \prec \frac{v^{\prime 2}}{\mu}, \frac{\partial \sigma}{\partial \rho} \prec 0$, with $\rho \succ \frac{v^{\prime 2}}{\mu}, \frac{\partial \sigma}{\partial \rho} \succ 0$. Let's analyze the behavior of the function $\sigma$. With $\rho \rightarrow 0, \sigma \rightarrow \infty$, with 
$\rho \rightarrow \infty, \sigma \rightarrow 0$. The more accurately determined right estimate for the function $\sigma$ is therefore written as:

$$
\begin{gathered}
\sigma \leq \sigma_{+}^{*}=\frac{\left(\gamma_{4} \cdot C^{*}\right)^{2}+\left(v^{\prime}+\gamma_{5} \cdot C^{*}\right)^{2}}{\left(\rho-\gamma_{1} \cdot C^{*}\right)^{2}}-\frac{2 \mu}{\rho-\gamma_{1} \cdot C^{*}}, \\
\sigma \geq \sigma_{-}^{*}=\frac{\left(v^{\prime}-\gamma_{5} \cdot C^{*}\right)^{2}}{\left(\rho+\gamma_{1} \cdot C^{*}\right)^{2}}-\frac{2 \mu}{\rho+\gamma_{1} \cdot C^{*}} .
\end{gathered}
$$

Then the majorant estimate for the function $\sigma$ is written as:

$$
\sigma^{*}=\max \left(\left|\sigma_{+}^{*}\right|,\left|\sigma_{-}^{*}\right|\right) \text {. }
$$

Taking into account the assumptions, let's determine the majorant estimates of the functions

$$
\begin{gathered}
x(u), y(u), x^{\prime}(u), y^{\prime}(u), \xi^{\prime \prime}(u), \eta^{\prime \prime}(u) . \\
x=\xi+\xi^{\prime} \cdot S_{1}+\xi^{\prime \prime} \cdot S_{2}, x \leq x_{+}^{*}=\xi+\gamma_{1} \cdot C^{*}+\gamma_{4} \cdot C^{*} \cdot S_{1}^{*}+\xi^{\prime \prime *} \cdot S_{2}^{*}, \\
x \geq x_{-}^{*}=\xi-\gamma_{1} \cdot C^{*}-\gamma_{4} \cdot C^{*} \cdot S_{1}^{*}-\xi^{\prime \prime *} \cdot S_{2}^{*}, \\
x^{*}=\max \left(\left|x_{+}^{*}\right|,\left|x_{-}^{*}\right|\right) .
\end{gathered}
$$

$S_{1}^{*}, S_{2}^{*}$ - majorant estimates of the Stumpff functions $S_{1}$ and $S_{2}$.

Functional series:

$$
S_{1}=\frac{u}{1 !}+\frac{\sigma \cdot u^{3}}{3 !}+\frac{\sigma^{2} \cdot u^{5}}{5 !}+\cdots
$$

is majorized by a series representing an infinitely decreasing geometric progression with the denominator $q=\frac{\sigma^{*} \cdot u_{1}^{2}}{6} \prec \prec 1$ of the form:

$$
S_{1} \leq S_{1}^{*}=\frac{u_{1}}{1 !} \cdot \frac{1}{1-\frac{\sigma^{*} \cdot u_{1}^{2}}{6}} .
$$

Majorizing series are similarly constructed for the remaining $S_{k}$.

$$
\begin{gathered}
S_{0} \leq S_{0}^{*}=\frac{1}{1-\frac{\sigma^{*} \cdot u_{1}^{2}}{2}}, \quad S_{2} \leq S_{2}^{*}=\frac{u_{1}^{2}}{2 !} \cdot \frac{1}{1-\frac{\sigma^{*} \cdot u_{1}^{2}}{12}}, \\
S_{3} \leq S_{3}^{*}=\frac{u_{1}^{3}}{3 !} \cdot \frac{1}{1-\frac{\sigma^{*} \cdot u_{1}^{2}}{20}}, \quad S_{4} \leq S_{4}^{*}=\frac{u_{1}^{4}}{4 !} \cdot \frac{1}{1-\frac{\sigma^{*} \cdot u_{1}^{2}}{30}}, \\
S_{5} \leq S_{5}^{*}=\frac{u_{1}^{5}}{5 !} \cdot \frac{1}{1-\frac{\sigma^{*} \cdot u_{1}^{2}}{42}}, \cdots
\end{gathered}
$$

Let's determine the majorant estimates of the remaining functions:

$$
\begin{aligned}
& \xi^{\prime \prime} \leq \xi_{+}^{\prime \prime *}=\frac{\left(v^{\prime}+\gamma_{5} \cdot C^{*}\right) \cdot \gamma_{4} \cdot C^{*}}{\rho-\gamma_{1} \cdot C^{*}}-\frac{\mu \cdot\left(\xi-\gamma_{1} \cdot C^{*}\right)}{\rho-\gamma_{1} \cdot C^{*}} \\
& \xi^{\prime \prime} \geq \xi_{-}^{\prime \prime^{*}}=\frac{\left(u^{\prime}-\gamma_{5} \cdot C^{*}\right) \cdot \gamma_{4} \cdot C^{*}}{\rho+\gamma_{1} \cdot C^{*}}-\frac{\mu \cdot\left(\xi-\gamma_{1} \cdot C^{*}\right)}{\rho+\gamma_{1} \cdot C^{*}}
\end{aligned}
$$




$$
\begin{gathered}
\eta^{\prime \prime} \leq \eta_{+}^{\prime \prime *}=\frac{\left(v^{\prime}+\gamma_{5} \cdot C^{*}\right)^{2}}{\rho-\gamma_{1} \cdot C^{*}}-\frac{\mu \cdot \gamma_{2} \cdot C^{*}}{\rho-\gamma_{1} \cdot C^{*}} \\
\eta^{\prime \prime} \geq \eta_{-}^{\prime \prime *}=\frac{\left(v^{\prime}-\gamma_{5} \cdot C^{*}\right)^{2}}{\rho+\gamma_{1} \cdot C^{*}}-\frac{\mu \cdot \gamma_{2} \cdot C^{*}}{\rho+\gamma_{1} \cdot C^{*}}, \\
y \leq y_{+}^{*}=\gamma_{2} \cdot C^{*}+\left(v^{\prime}+\gamma_{5} \cdot C^{*}\right) \cdot S_{1}^{*}+\eta^{\prime \prime *} \cdot S_{2}^{*} \\
y \geq y_{-}^{*}=-\gamma_{2} \cdot C^{*}+\left(v^{\prime}-\gamma_{5} \cdot C^{*}\right) \cdot S_{1}^{*}-\eta^{\prime \prime *} \cdot S_{2}^{*} \\
x^{\prime} \leq x_{+}^{\prime *}=\gamma_{4} \cdot C^{*} \cdot S_{0}^{*}+\xi^{\prime \prime *} \cdot S_{1}^{*} ; x^{\prime} \geq x_{-}^{\prime *}=-\gamma_{4} \cdot C^{*} \cdot S_{0}^{*}-\xi^{\prime \prime *} \cdot S_{1}^{*}, \\
y^{\prime} \leq y_{+}^{\prime *}=\left(v^{\prime}+\gamma_{5} \cdot C^{*}\right) \cdot S_{0}^{*}+\eta^{\prime \prime *} \cdot S_{1}^{*}, y^{\prime} \geq y_{-}^{\prime *}=\left(v^{\prime}-\gamma_{5} \cdot C^{*}\right) \cdot S_{0}^{*}-\eta^{\prime \prime *} \cdot S_{1}^{*}, \\
\xi^{\prime \prime *}=\max \left(\left|\xi_{+}^{\prime \prime}\right|,\left|\xi_{-}^{\prime \prime}\right|\right), \quad \eta^{\prime \prime *}=\max \left(\left|\eta_{+}^{\prime \prime *}\right|,\left|\eta_{-}^{\prime \prime}\right|\right), \quad y^{*}=\max \left(\left|y_{+}^{*}\right|,\left|y_{-}^{*}\right|\right), \\
x^{\prime *}=\max \left(\left|x_{+}^{\prime *}\right|,\left|x_{-}^{\prime *}\right|\right), \quad y^{\prime *}=\max \left(\left|y_{+}^{\prime *}\right|,\left|y_{-}^{\prime^{*}}\right|\right) .
\end{gathered}
$$

Let's determine the majorant estimates for perturbing acceleration constituents $X, Y$.

$$
\begin{aligned}
X \leq X_{+}^{*} & =\frac{\mu}{\Delta_{1 \min }^{2}} \cdot\left|\frac{x_{1}-x}{\Delta_{1}}\right|+\frac{\mu_{1}}{a_{1}^{2}} \cdot\left|\frac{x_{1}}{a_{1}}\right| \leq \frac{\mu_{1}}{\Delta_{1 \min }^{2}}+\frac{\mu_{1}}{a_{1}^{2}}, \\
X & \geq X_{-}^{*} \geq \frac{\mu_{1}}{\Delta_{1 \max }^{2}}+\frac{\mu_{1}}{a_{1}^{2}}, X^{*}=\max \left(\left|X_{+}^{*}\right|,\left|X_{-}^{*}\right|\right),
\end{aligned}
$$

where: $\Delta_{1 \max }^{2}=\left(a_{1}+x^{*}\right)^{2}+\left(a_{1}+y^{*}\right)^{2}, \Delta_{1 \min }^{2}=\left(x_{10}-x^{*}\right)^{2}+\left(y_{10}-y^{*}\right)^{2}$,

$$
x_{10}=a_{1} \cdot \cos \theta_{10}, \quad y_{10}=a_{1} \cdot \sin \theta_{10} .
$$

The estimate for the function $Y(u)$. is determined similarly. Thus, the quantity $A_{1}$ is estimated as follows

$$
A_{1}=\alpha_{1}^{*} \cdot X^{*}+\beta_{1}^{*} \cdot Y^{*}
$$

where $\alpha_{1}^{*}, \beta_{1}^{*}$ are majorant estimates of the functions $\alpha_{1}(u), \beta_{1}(u)$ obtained in the paper [3]. Similar operations are made for other system equations [2]. Among the quantities $A_{i}(i=1,2, \ldots, 7)$. the maximum one is chosen as the quantity A. The quantity $B_{1}$ is estimated as follows:

$$
B_{1}=n \cdot\left(\frac{\partial \alpha_{1}^{*}}{\partial \xi} \cdot X^{*}+\frac{\partial \beta_{1}^{*}}{\partial \xi} \cdot Y^{*}+\alpha_{1}^{*} \cdot \frac{\partial X^{*}}{\partial \xi}+\beta_{1}^{*} \cdot \frac{\partial Y^{*}}{\partial \xi}\right)
$$

Where: $\frac{\partial \alpha_{1}(u)}{\partial \xi}=-\frac{\partial r}{\partial \xi} \cdot\left(\rho \cdot S_{1}+\rho^{\prime} \cdot S_{2}\right)-r\left(\frac{\partial \rho}{\partial \xi} S_{1}+\rho^{\prime} \cdot \frac{\partial S_{2}}{\partial \sigma} \cdot \frac{\partial \sigma}{\partial \xi}+\frac{\partial \rho^{\prime}}{\partial \xi} S_{2}+\rho \cdot \frac{\partial S_{1}}{\partial \sigma} \cdot \frac{\partial \sigma}{\partial \xi}\right)+$

$$
\begin{gathered}
+\frac{\partial S_{2}}{\partial \sigma} \cdot \frac{\partial \sigma}{\partial \xi} x^{\prime} \cdot \xi+S_{2} \frac{\partial x^{\prime}}{\partial \xi} \cdot \xi+S_{2} \cdot x^{\prime}+\frac{\partial S_{3}}{\partial \sigma} \frac{\partial \sigma}{\partial \xi} \xi^{\prime} x^{\prime}+S_{3} \frac{\partial x^{\prime}}{\partial \xi} \cdot \xi^{\prime} ; \\
\frac{\partial X(u)}{\partial \xi}=\frac{2 \mu}{\Delta_{1}^{3}(u)} \cdot\left(\frac{\partial x(u)}{\partial \xi}+\frac{\partial y(u)}{\partial \xi}\right),
\end{gathered}
$$

$n$ - number of system equations. $\frac{\partial \beta_{1}(u)}{\partial \xi}, \frac{\partial Y(u)}{\partial \xi}$. are determined similarly. In the expression (25), the quantities $\frac{\partial \alpha_{1}^{*}}{\partial \xi}, \frac{\partial \beta_{1}^{*}}{\partial \xi}$ are the majorant estimates of the functions $\frac{\partial \alpha_{1}(u)}{\partial \xi}, \frac{\partial \beta_{1}(u)}{\partial \xi}$, respectively. To determine them, it is necessary to find the majorant estimates of the 
functions being part of the expression (26). Below some majorant estimates of these functions are shown.

$$
\begin{aligned}
& \frac{\partial r(u)}{\partial \xi}=\frac{x \cdot \frac{\partial x}{\partial \xi}+y \cdot \frac{\partial y}{\partial \xi}}{r}, \\
& \frac{\partial x}{\partial \xi}=1+\xi^{\prime} \cdot \frac{\partial S_{1}}{\partial \sigma} \cdot \frac{\partial \sigma}{\partial \xi}+\xi^{\prime \prime} \cdot \frac{\partial S_{2}}{\partial \sigma} \cdot \frac{\partial \sigma}{\partial \xi}+S_{2} \cdot \frac{\partial \xi^{\prime \prime}}{\partial \xi}, \\
& \frac{\partial \xi^{\prime \prime}}{\partial \xi}=\frac{\frac{\partial \rho^{\prime}}{\partial \xi} \cdot \xi^{\prime} \cdot \rho+\mu \xi \cdot \frac{\partial \rho}{\partial \xi}-\frac{\partial \rho}{\partial \xi} \cdot \rho^{\prime} \cdot \xi^{\prime}-\mu \rho}{\rho^{2}}, \\
& \frac{\partial S_{1}}{\partial \sigma} \leq \frac{\partial S_{1}^{*}}{\partial \sigma}=\frac{u_{1}^{3}}{6\left(1-\frac{\sigma^{*} u_{1}^{2}}{6}\right)^{2}}, \quad \frac{\partial S_{3}}{\partial \sigma} \leq \frac{\partial S_{3}^{*}}{\partial \sigma}=\frac{u_{1}^{5}}{120\left(1-\frac{\sigma^{*} u_{1}^{2}}{20}\right)^{2}}, \\
& \frac{\partial S_{2}}{\partial \sigma} \leq \frac{\partial S_{2}^{*}}{\partial \sigma}=\frac{u_{1}^{4}}{24\left(1-\frac{\sigma^{*} u_{1}^{2}}{12}\right)^{2}}, \quad \frac{\partial S_{0}}{\partial \sigma} \leq \frac{\partial S_{0}^{*}}{\partial \sigma}=\frac{u_{1}^{2}}{2\left(1-\frac{\sigma^{*} u_{1}^{2}}{2}\right)^{2}}, \\
& \frac{\partial S_{4}}{\partial \sigma} \leq \frac{\partial S_{4}^{*}}{\partial \sigma}=\frac{u_{1}^{6}}{720\left(1-\frac{\sigma^{*} u_{1}^{2}}{30}\right)^{2}}, \frac{\partial S_{5}}{\partial \sigma} \leq \frac{\partial S_{5}^{*}}{\partial \sigma}=\frac{u_{1}^{7}}{5040\left(1-\frac{\sigma^{*} u_{1}^{2}}{42}\right)^{2}} \text {, } \\
& \frac{\partial x^{\prime}}{\partial \xi}=\xi^{\prime} \frac{\partial S_{0}}{\partial \sigma} \cdot \frac{\partial \sigma}{\partial \xi}+\xi^{\prime \prime} \frac{\partial S_{1}}{\partial \sigma} \cdot \frac{\partial \sigma}{\partial \xi}+S_{1} \cdot \frac{\partial \xi^{\prime \prime}}{\partial \xi} \\
& \left|\frac{\partial \rho}{\partial \xi}\right| \leq 1, \frac{\partial \rho^{\prime}}{\partial \xi} \leq \frac{\partial \rho^{\prime *}}{\partial \xi_{+}}=\frac{v^{\prime}+2 \gamma_{3} \cdot C^{*}}{\rho-\gamma_{1} C^{*}}, \quad \frac{\partial \rho^{\prime}}{\partial \xi} \geq \frac{\partial \rho^{\prime^{*}}}{\partial \xi_{-}}=\frac{{v^{\prime}-2 \gamma_{3} \cdot C^{*}}^{*}+\gamma_{1} C^{*}}{,} \\
& \frac{\partial \sigma}{\partial \xi} \leq \frac{\partial \sigma^{*}}{\partial \xi_{+}}=\frac{-2\left(v^{\prime}+\gamma_{5} C^{*}\right)^{2}}{\left(\rho-\gamma_{1} C^{*}\right)^{3}}+\frac{2 \mu}{\left(\rho-\gamma_{1} C^{*}\right)^{2}}, \quad \frac{\partial \sigma}{\partial \xi} \geq \frac{\partial \sigma^{*}}{\partial \xi_{-}}=\frac{-2\left(v^{\prime}-\gamma_{5} C^{*}\right)^{2}}{\left(\rho+\gamma_{1} C^{*}\right)^{3}}+\frac{2 \mu}{\left(\rho+\gamma_{1} C^{*}\right)^{2}} \text {. }
\end{aligned}
$$

Similar operations were made for other equations of the perturbed motion differential equations system [2]. As a result, the quantity $B$. is estimated. Thus, we can estimate the parameter quantity $C^{*}$ using the expression obtained in the paper [3]. Due to the large amount of calculations, the algorithm is implemented for computer calculations.

\section{An example of successive approximation convergence domain estimation}

To illustrate the developed technique of studying successive approximations convergence, the calculations were made with given initial conditions for the body parabolic motion taking into account the assumptions and a numerical estimate of the convergence domain was obtained. Several variants were calculated with different values of independent variable $u$ from $C^{*}$ and it was shown that this dependence had an extremum. Dependence of the parameter $C^{*}$ on the quantity of the scale coefficients $\gamma_{i},(i=1,2,4,5,7)$ was analyzed. It is shown that in degenerate case with $\gamma_{i}=0$ the dependence of the quantity $C^{*}$ on the variable $u$ has no extremum. When increasing the coefficients $\gamma_{i}$, this dependence has an extremum, which indicates an extension of the convergence domain. But the quantity $C^{*}$ is 
limited, therefore we should state a certain range of scale coefficients, where the extremum of the function $C^{*}$ does not exceed a certain value. When giving the value of the parameter $C^{*}=10$, it was defined more accurately using the developed technique. For the given initial conditions, we found the extremal value of the quantity $C^{*}=4.9871$ and the corresponding value of the independent variable $u_{1}=0.24$. Thus, if the mismatches in the rectangular coordinates of a perturbed body motion at the same instants of time at different stages of approximation do not exceed the quantity $C^{*}=4.9871$, the successive approximations which represent the solution of the perturbed motion differential equations system converge absolutely.

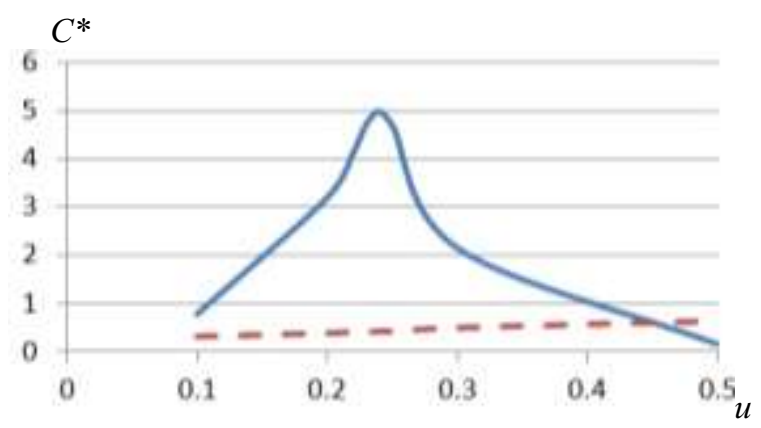

Fig. 1. The dependence of the parameter $C^{*}$ on the normalized regularizing variable $u$

\section{Conclusions}

A research method for the convergence of Picard's successive approximations, representing a solution of the perturbed body motion differential equations system, was developed. A method for finding majorizing function estimates, required for determination of the successive approximations convergence, was developed. These estimates were determined and analytical formulas for determination of the convergence domain boundaries were produced.

The developed technique for study of the Picard's successive approximations convergence, which represents the solution of the perturbed body motion differential equations system, can be used in underground construction.

\section{References}

1. G. Merman, Bulletin Theor. Astronomy Inst., 6, 713-731 (1958)

2. V. Petelina, Developm. of astronomy research meth., 8, 247-258 (1979)

3. V. Petelina, MATEC Web Conf. 117, 00135 (2017) 